Journal of Engineering and Applied Sciences 14 (13): 4405-4413, 2019

ISSN: 1816-949X

(C) Medwell Journals, 2019

\title{
Analysis of Pressure-Driven Heat and Mass Transfer of Hydromagnetic Flow Past Darcy-Forchheimer Porous Media Using Lie Group
}

\author{
${ }^{1}$ S.O. Salawu, ${ }^{2}$ M.S. Dada and ${ }^{1}$ O. Adebimpe \\ ${ }^{1}$ Department of Mathematics, Landmrk University, Omu-Aran, Nigeria \\ ${ }^{2}$ Department of Mathematics, University of Ilorin, Ilorin, Nigeria
}

\begin{abstract}
This study investigates heat and mass transfer of Magnetohydrodynamic (MHD) pressure-driven flow in a Darcy-Forchheimer porous medium with inclined uniform magnetic field and thermal radiation. The governing equations of the model are reduced to a system of coupled non-linear ordinary differential equations by applying a scaling group of transformations. The coupled differential equations are then solved using Weighted Residual Method (WRM) with the results compared with that obtained from shooting technique coupled with fourth order Runge-Kutta method. The obtained results are presented graphically to represent the effect various fluid parameters on the dimensionless velocity, temperature, concentration and pressure drop. Finally, the effects of Skin friction, Nusselt and Sherwood numbers which are of physical and engineering interest are presented and discussed.
\end{abstract}

Key words: Magnetohydrodynamic, pressure-driven flow, porous medium, Darcy-Forchheimer, weighted residual method, thermal radiation

\section{INTRODUCTION}

The study of MHD fluid flow induced by the concurrent actions of buoyancy forces follow-on from the mass diffusion and thermal conductivity is gaining attention due to its interesting applications. The radiative thermal physics of MHD problems with heat and mass transfer through Darcy-Forchheimer permeable medium are usually come across in the power engineering and other pollutant, solidification and evaporation cooling, grain storage are areas in which the combined thermo-solutal in a permeable medium are experienced.

In few of its applications, Singh et al. (2012) examined heat and mass transfer in MHD flow through a vertical stretching surface in the occurrence of heat generation. Analysis of the MHD stagnation point fluid flow with the effects of chemical reaction on heat and mass transfer near a permeable stretching vertical sheet and injection or suction by Lie group was investigated by Afify and Elgazery (2012). The result presents monotonically decreased when the magnetic field parameter was increased. Alam et al. (2003) reported on viscous dissipative effect of $\mathrm{MHD}$ convective flow over an inclined permeable stretching sheet and chemical reaction. It was found that chemical reaction may significantly influenced the concentration boundary layer and wall mass transfer rate while Ahmed and Bhattacharyya (2014) studied free convection chemical reaction in MHD couette flow with heat source. The above researchers did not consider the effects of pressure gradient, porosity and radiation on the fluid flow.

The analysis of flow past porous medium has received the attention of several researchers due to its extensive applications in enhancing recovery of petroleum, chemical engineering, etc. The thermal diffusion and heat generation of heat and mass transfer in MHD fluid flow through a vertical motioning permeable sheet and medium was examined by Saxena and Dubey (2011) while Hunegnaw and Kishan (2014) examined heat and mass transfer in a MHD flow along a moving surface with chemical reaction, variable properties in a porous medium and viscous dissipation. The cited researchers did not consider the significant of non-Darcy porous medium and radiation on the flow as well as the influence of fluid parameters on the pressure.

In studies of heat and mass transfer, Ishak and Nazar (2006) analyzed boundary layers of a permeable medium over a vertical surface by applying Brinkman equation for the porous medium model while Loganathan and Arasu (2010) investigated thermophoresis influences on heat and mass transfer in a MHD Darcy-Forchheimer permeable medium in the occurrence injection/suction over a porous wedge. It was noticed that the flow velocity decreased as the inertial term increased, the behavior was due to the fact that the inertia parameter supports the resistance of the flow fluid mechanism. Also,

Corresponding Author: S.O. Salawu, Department of Mathematics, Landmrk University, Omu-Aran, Nigeria 
Ahammad et al. (2013) examined heat and mass transfer through a non-Darcy permeable medium in the presence of magnetic field and (Hemalatha et al., 2014) investigated thermal dispersion with melting on Darcy-Forchheimer porous medium in a hydromagnetic flow over a stretching surface. Forchheimer extension for the flow equations in steady state was considered.

Moreover, Lee et al. (2008) examined radiative heat transfer past a porous sheet using Lie group. It was obtained that the heat and velocity boundary layer thicknesses increased by a rise in the radiation term, variational increased in the Prandtl number slow down both the temperature and velocity in the boundary layer. Velocity increased and temperature decreased when there was an increased in Grashof number and the porosity parameter. Chauhan and Rastogi (2010) analyzed natural convection effects on radiative MHD flow through a vertical partially filled porous medium without giving attention to the effect of mass diffusion and heat generation in the studied. Dada and Adefolaju (2012) reported on hydrodynamic flow fluid over a boundary layer in a stretching sheet entrenched in a radiation and non-Darcy permeable medium. It was found that an increase in the Forchheimer number reduced the velocity profile. However, the study did not includes the effect of pressure gradient, heat source and chemical reaction rate on the flow while Kumar and Sivaraj (2012) carried out analysis on viscoelastic fluid of $\mathrm{MHD}$ flow in non-Darcy permeable medium along a motioning vertical conduit.

Follow from the above studies, the researchers neglected the influence of Darcy-Forchheimer permeable medium inclined magnetic field and thermal radiation on a fluid flow as well as the effects of fluid parameters on the pressure. The current study examine the combined influences of inclined magnetic field, pressure drop, thermal radiation and Darcy-Forchheimer porous medium in a steady convective heat and mass transfer in a MHD flow. The fluid flow is pressure-driven past a permeable plate with inclined uniform magnetic field.

\section{MATERIALS AND METHODS}

Formulation of the problem: Examine heat and mass transfer of MHD pressure-driven, steady, viscous, laminar and incompressible fluid over a permeable stretching plate in Darcy-Forchheimer medium and radiation under the effect of inclined magnetic field and pressure gradient. The fluid motion is maintained by both pressure gradient and gravity and the flow is assumed to be in the direction of $\mathrm{X}$ and $\mathrm{Y}$-axis normal to it. A uniform magnetic field $\mathrm{B}_{0}$ is introduced at angle $\alpha$ lying in the range $0<\alpha<\pi / 2$ in the

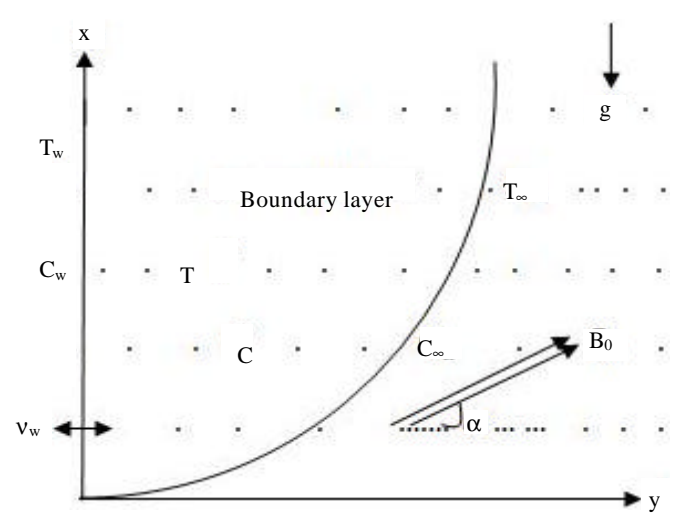

Fig. 1: Geometry of the problem

direction of the flow. The plate is maintained at the temperature and species concentration $\mathrm{T}_{\mathrm{w}} \mathrm{C}_{\mathrm{w}}$ and free stream temperature and species concentration $\mathrm{T}_{\infty} \mathrm{C}_{\infty}$, respectively. The equations governing the steady radiative heat and mass transfer of two-dimensional magnetohydrodynamics pressure-driven fluid flow in Darcy Forchheimer porous medium with inclined magnetic field are as follows (Fig. 1):

$$
\begin{gathered}
\frac{\partial \mathrm{U}}{\partial \mathrm{X}}+\frac{\partial \mathrm{V}}{\partial \mathrm{Y}}=0 \\
\mathrm{U} \frac{\partial \mathrm{U}}{\partial \mathrm{X}}+\mathrm{V} \frac{\partial \mathrm{U}}{\partial \mathrm{Y}}=-\frac{1}{\rho} \sigma \mathrm{B}_{0}^{2} \mathrm{U} \sin ^{2} \alpha-\frac{1}{\rho} \frac{\partial \mathrm{P}}{\partial \mathrm{X}}+ \\
v\left(\frac{\partial^{2} \mathrm{U}}{\partial \mathrm{X}^{2}}+\frac{\partial^{2} \mathrm{U}}{\partial \mathrm{Y}^{2}}\right)-\frac{v}{\mathrm{~K}^{*}} \mathrm{U}-\frac{\mathrm{b}}{\mathrm{K}^{*}} \mathrm{U}^{2}+ \\
\mathrm{g} \beta_{\mathrm{T}}\left(\mathrm{T}-\mathrm{T}_{\infty}\right)+\mathrm{g} \beta_{\mathrm{C}}\left(\mathrm{C}-\mathrm{C}_{\infty}\right) \\
\mathrm{U} \frac{\partial \mathrm{V}}{\partial \mathrm{X}}+\mathrm{V} \frac{\partial \mathrm{V}}{\partial \mathrm{Y}}=-\frac{1}{\rho} \frac{\partial \mathrm{P}}{\partial \mathrm{Y}}+v\left(\frac{\partial^{2} \mathrm{~V}}{\partial \mathrm{X}^{2}}+\frac{\partial^{2} \mathrm{~V}}{\partial \mathrm{Y}^{2}}\right) \\
\rho \mathrm{C}_{\mathrm{p}}\left(\mathrm{U} \frac{\partial \mathrm{T}}{\partial \mathrm{X}}+\mathrm{V} \frac{\partial \mathrm{T}}{\partial \mathrm{Y}}\right)=\mathrm{k}\left(\frac{\partial^{2} \mathrm{~T}}{\partial \mathrm{X}^{2}}+\frac{\partial^{2} \mathrm{~T}}{\partial \mathrm{Y}^{2}}\right)- \\
\frac{\partial \mathrm{q}_{\mathrm{X}}}{\partial \mathrm{X}}-\frac{\partial \mathrm{q}_{\mathrm{Y}}}{\partial \mathrm{Y}}+\mathrm{Q}_{0}\left(\mathrm{~T}-\mathrm{T}_{\infty}\right) \\
\mathrm{\partial C}+\mathrm{V} \frac{\partial \mathrm{C}}{\partial \mathrm{Y}}=\mathrm{D}\left(\frac{\partial^{2} \mathrm{C}}{\partial \mathrm{X}^{2}}+\frac{\partial^{2} \mathrm{C}}{\partial \mathrm{Y}^{2}}\right)-\gamma\left(\mathrm{C}-\mathrm{C}_{\infty}\right)
\end{gathered}
$$

The corresponding initial and boundary conditions are as follows: 


$$
\begin{aligned}
& \mathrm{U}=0, \mathrm{~V}=v_{\mathrm{w}}, \mathrm{P}=0, \mathrm{~T}=\mathrm{T}_{\infty}+\left(\mathrm{T}_{\mathrm{w}}-\mathrm{T}_{\infty}\right) \mathrm{AX}, \\
& \mathrm{C}=\mathrm{C}_{\infty}+\left(\mathrm{C}_{\mathrm{w}}-\mathrm{C}_{\infty}\right) \mathrm{BX} \text { at } \mathrm{Y}=0 \\
& \mathrm{U}=0, \mathrm{~T}=\mathrm{T}_{\infty}, \mathrm{C}=\mathrm{C}_{\infty} \text { as } \mathrm{Y} \rightarrow \infty
\end{aligned}
$$

where, $\mathrm{U}, \mathrm{V}, \mathrm{P}, \mathrm{C}$ and $\mathrm{T}$ are the velocity component in the $\mathrm{X}$ direction, velocity component in the $\mathrm{Y}$ direction, pressure, concentration of species in the fluid and temperature of the fluid, respectively. $\mathrm{A}$ and $\mathrm{B}$ are constants defined as $\mathrm{A}=\mathrm{B}=1 / 1, \mathrm{~B}_{0}$ is the magnetic field strength, $\alpha$ is the angle of inclination of the magnet, $v_{\mathrm{w}}$ is the permeability of the porous surface such that $v_{\mathrm{w}}>0$ indicates wall injection and $v_{\mathrm{w}}<0$ indicates wall suction, respectively. The physical quantities $v, b, K^{*}, \rho, \alpha, D, C_{p}$, $\mathrm{k}, \mathrm{Q}_{0}$ and $\gamma$ are the fluid kinematics viscosity, Forchheimer parameter of the medium, permeability of the porous medium, density, electric conductivity of the fluid, mass diffusion coefficient, specific heat at constant pressure, thermal conductivity, internal heat generation or absorption and chemical reaction coefficient, respectively. $\mathrm{g}$ is the gravitational acceleration, $\beta_{\mathrm{T}}$ and $\beta_{\mathrm{C}}$ are the thermal and concentration expansion coefficients, respectively. $q_{x}$ and $q_{y}$ are the radiative heat flux in the $X$ and $\mathrm{Y}$ direction, respectively. Using Rosseland diffusion approximation for radiation by Kareem et al. (2018):

$$
\mathrm{q}_{\mathrm{X}}=-\frac{4 \sigma_{0}}{3 \delta} \frac{\partial \mathrm{T}^{4}}{\partial \mathrm{X}} \text { and } \mathrm{q}_{\mathrm{Y}}=-\frac{4 \sigma_{0}}{3 \delta} \frac{\partial \mathrm{T}^{4}}{\partial \mathrm{Y}}
$$

where, $\sigma_{0}$ and $\delta$ are the Stefan-Boltzmann and the mean absorption coefficient, respectively. Assume the temperature difference within the flow are sufficiently small such that $\mathrm{T}^{4}$ may be expressed as a linear function of temperature, using Taylor series to expand $\mathrm{T}^{4}$ about the free stream $\mathrm{T}_{\infty}$ and neglecting higher order terms, this gives the approximation:

$$
\mathrm{T}^{4} \cong 4 \mathrm{~T}_{\infty}^{3} \mathrm{~T}-3 \mathrm{~T}_{\infty}^{4}
$$

Using Eq. 8 then, Eq. 7 can be express as:

$$
\frac{\partial \mathrm{q}_{\mathrm{x}}}{\partial \mathrm{X}}=-\frac{16 \sigma_{0} \mathrm{~T}_{\infty}^{3}}{3 \delta} \frac{\partial^{2} \mathrm{~T}}{\partial \mathrm{X}^{2}} \text { and } \frac{\partial \mathrm{q}_{\mathrm{Y}}}{\partial \mathrm{Y}}=-\frac{16 \sigma_{0} \mathrm{~T}_{\infty}^{3}}{3 \delta} \frac{\partial^{2} \mathrm{~T}}{\partial \mathrm{Y}^{2}}
$$

Introducing the following non-dimensional quantities:

$$
\begin{gathered}
\mathrm{x}=\frac{\mathrm{X}}{1}, \mathrm{y}=\frac{\mathrm{Y}}{1}, \mathrm{u}=\frac{\mathrm{U} 1}{v}, v=\frac{\mathrm{V} 1}{v}, \mathrm{p}=\frac{\mathrm{Pl}^{2}}{\rho v^{2}}, \\
\phi=\frac{\mathrm{C}-\mathrm{C}_{\infty}}{\mathrm{C}_{\mathrm{w}}-\mathrm{C}_{\infty}}, \theta=\frac{\mathrm{T}-\mathrm{T}_{\infty}}{\mathrm{T}_{\mathrm{w}}-\mathrm{T}_{\infty}}
\end{gathered}
$$

Substituting (Eq. 9) and (Eq. 10) into Eq. 1-6, to obtain:

$$
\begin{gathered}
\frac{\partial \mathrm{u}}{\partial \mathrm{x}}+\frac{\partial \mathrm{v}}{\partial \mathrm{y}}=0 \\
\mathrm{u} \frac{\partial \mathrm{u}}{\partial \mathrm{x}}+v \frac{\partial \mathrm{u}}{\partial \mathrm{y}}=-\mathrm{H}_{\mathrm{a}}^{2} \sin ^{2} \alpha \mathrm{u}-\frac{\partial \mathrm{p}}{\partial \mathrm{x}}+ \\
\left.\frac{\partial^{2} \mathrm{u}}{\partial \mathrm{x}^{2}}+\frac{\partial^{2} \mathrm{u}}{\partial \mathrm{y}^{2}}\right)-\mathrm{D}_{\mathrm{a}} \mathrm{u}-\mathrm{F}_{\mathrm{s}} \mathrm{u}^{2}+\mathrm{G}_{\mathrm{r}} \theta+\mathrm{G}_{\mathrm{c}} \phi \\
\mathrm{u} \frac{\partial v}{\partial \mathrm{x}}+v \frac{\partial v}{\partial \mathrm{y}}=-\frac{\partial \mathrm{p}}{\partial \mathrm{y}}+\left(\frac{\partial^{2} v}{\partial \mathrm{x}^{2}}+\frac{\partial^{2} v}{\partial \mathrm{y}^{2}}\right) \\
+v \frac{\partial \theta}{\partial \mathrm{y}}=\frac{1}{\mathrm{P}_{\mathrm{r}}}\left(1+\frac{4}{3} \mathrm{R}\right)\left(\frac{\partial^{2} \theta}{\partial \mathrm{x}^{2}}+\frac{\partial^{2} \theta}{\partial \mathrm{y}^{2}}\right)+\mathrm{Q} \theta \\
\mathrm{u} \frac{\partial \phi}{\partial \mathrm{x}}+v \frac{\partial \phi}{\partial \mathrm{y}}=\frac{1}{\mathrm{~S}}\left(\frac{\partial^{2} \phi}{\partial \mathrm{x}^{2}}+\frac{\partial^{2} \phi}{\partial \mathrm{y}^{2}}\right)-\lambda \phi
\end{gathered}
$$

The corresponding initial and boundary conditions are follows:

$$
\begin{gathered}
u=0, v=-f_{w}, p=0, \theta=x, \phi=x \text { at } y=0 \\
u=0, \theta=0, \phi=0 \text { as } y \rightarrow \infty
\end{gathered}
$$

where, the term $\mathrm{P}_{\mathrm{r}}=\mu \mathrm{C}_{\mathrm{p}} / \mathrm{k}$ is the Prandtl, $\mathrm{Sc}=v / \mathrm{D}$ is the Schmidt, $\mathrm{H}_{\mathrm{a}}=\mathbb{B}_{0} \sqrt{\sigma / \mu}$ is the Hartmann, $\mathrm{G}_{\mathrm{r}}=1^{3} \mathrm{~g} \beta_{\mathrm{T}}\left(\mathrm{T}_{\mathrm{w}}-\mathrm{T}_{\infty} / v^{2}\right)$ is the thermal Grashof, $\mathrm{G}_{\mathrm{c}}=1^{3} \mathrm{~g} \beta_{\mathrm{T}}\left(\mathrm{C}_{\mathrm{w}}-\mathrm{C}_{\mathrm{o}} / v^{2}\right)$ is the solutal Grashof, $Q=1^{2} Q_{0} / \mu C_{p}$ is the heat source, $\lambda=1^{2} \gamma / \nu$ is the concentration parameter. $\mathrm{D}_{\mathrm{a}}=1^{2} / \mathrm{K}^{*}$ is the Darcy parameter, $\mathrm{F}_{\mathrm{s}}=\mathrm{la} / \mathrm{K}^{*}$ is the Forchheimer inertia term, $\mathrm{R}=$ $4 \sigma_{0} \mathrm{~T}^{3} / \delta \mathrm{k}$ is the Radiation heat transfer parameter and $\mathrm{f}_{\mathrm{w}}=v_{\mathrm{w}} 1 / v$ is the non-dimensional wall mass transfer coefficient, respectively.

Introducing the stream function, $\mathrm{u}=\partial \psi / \partial \mathrm{y}, v=\partial \psi / \partial \mathrm{x}$ continuity equation is automatically satisfied and Eq. 12-16 becomes:

$$
\begin{gathered}
\frac{\partial \psi}{\partial \mathrm{y}} \frac{\partial^{2} \psi}{\partial \mathrm{x} \partial \mathrm{y}}-\frac{\partial \psi}{\partial \mathrm{x}} \frac{\partial^{2} \psi}{\partial \mathrm{y}^{2}}=-\mathrm{H}_{\mathrm{a}}^{2} \sin ^{2} \alpha\left(\frac{\partial \psi}{\partial \mathrm{y}}\right)-\frac{\partial \mathrm{p}}{\partial \mathrm{x}}+ \\
\left(\frac{\partial^{3} \psi}{\partial \mathrm{x}^{2} \partial \mathrm{y}}+\frac{\partial^{3} \psi}{\partial \mathrm{y}^{3}}\right)-\mathrm{D}_{\mathrm{a}}\left(\frac{\partial \psi}{\partial \mathrm{y}}\right)-\mathrm{F}_{\mathrm{s}}\left(\frac{\partial \psi}{\partial \mathrm{y}}\right)^{2}+\mathrm{G}_{\mathrm{r}} \theta+\mathrm{G}_{\mathrm{c}} \phi \\
-\frac{\partial \psi}{\partial \mathrm{y}} \frac{\partial^{2} \psi}{\partial \mathrm{x}^{2}}+\frac{\partial \psi}{\partial \mathrm{x}} \frac{\partial^{2} \psi}{\partial \mathrm{x} \partial \mathrm{y}}=-\frac{\partial \mathrm{p}}{\partial \mathrm{y}}-\left(\frac{\partial^{3} \psi}{\partial \mathrm{x}^{3}}+\frac{\partial^{3} \psi}{\partial \mathrm{x}^{2} \mathrm{y}^{2}}\right)
\end{gathered}
$$




$$
\begin{gathered}
\frac{\partial \psi}{\partial \mathrm{y}} \frac{\partial \theta}{\partial \mathrm{x}}-\frac{\partial \psi}{\partial \mathrm{x}} \frac{\partial \theta}{\partial \mathrm{y}}=\frac{1}{\mathrm{P}_{\mathrm{r}}}\left(1+\frac{4}{3} \mathrm{R}\right)\left(\frac{\partial^{2} \theta}{\partial \mathrm{x}^{2}}+\frac{\partial^{2} \theta}{\partial \mathrm{y}^{2}}\right)+\mathrm{Q \theta} \\
\frac{\partial \psi}{\partial \mathrm{y}} \frac{\partial \phi}{\partial \mathrm{x}}-\frac{\partial \psi}{\partial \mathrm{x}} \frac{\partial \phi}{\partial \mathrm{y}}=\frac{1}{\mathrm{~S}_{\mathrm{c}}}\left(\frac{\partial^{2} \phi}{\partial \mathrm{x}^{2}}+\frac{\partial^{2} \phi}{\partial \mathrm{y}^{2}}\right)-\lambda \phi
\end{gathered}
$$

Subject to the initial and boundary conditions:

$$
\begin{aligned}
& \frac{\partial \psi}{\partial y}=0, \frac{\partial \psi}{\partial x}=f_{w}, p=0, \theta=x, \phi=x \text { at } y=0 \\
& \frac{\partial \psi}{\partial y}=0, \theta=0, \phi=0 \text { as } y \rightarrow \infty
\end{aligned}
$$

Introducing simplified form of Lie-group transformations namely, the scaling group of transformations to Eq. 17-21 which is equivalent to determining the invariant solutions of these equations under a continuous one-parameter group according to Pramanik (2013). One of the methods is to search for a transformation group from an elementary set of one-parameter scaling group of transformations, given as $\nabla$ :

$$
\begin{aligned}
& \nabla: \mathrm{x}^{*}=\mathrm{xe}^{\varepsilon \alpha_{1}}, \mathrm{y}^{*}=\mathrm{ye}^{\varepsilon \alpha_{2}}, \psi^{*}=\psi \mathrm{e}^{\varepsilon \alpha_{3}}, \\
& \mathrm{u}^{*}=\mathrm{ue}^{\varepsilon \alpha_{4}}, \nu^{*}=v \mathrm{e}^{\varepsilon \alpha_{5}}, \\
& \mathrm{p}^{*}=\mathrm{pe}^{\varepsilon \alpha_{6}}, \theta^{*}=\theta \mathrm{e}^{\varepsilon \alpha_{7}}, \phi^{*}=\phi \mathrm{e}^{\varepsilon \alpha_{8}}
\end{aligned}
$$

where, $\alpha_{1}-\alpha_{8}$ are transformation parameters of the group to be determined later and $\varepsilon$ is a small parameters. The task is to find relationships among the exponents $\alpha$ 's such that (Eq. 17-21) will remain invariant under the point transformations. Substituting transformation (Eq. 22) into Eq. 17-21 and applying invariant conditions yields the similarity transformations:

$$
\begin{gathered}
\eta=y, \psi=x f(\eta), p=p_{d}(\eta), \\
\theta=x \theta(\eta), \phi=x \phi(\eta)
\end{gathered}
$$

Substituting, the similarity variables (Eq. 23) into Eq. 17-21. The following system of non-linear differential equations are obtained:

$$
\begin{gathered}
\mathrm{f}^{\prime \prime \prime}+\mathrm{ff}^{\prime \prime}-\left(1+\mathrm{F}_{\mathrm{s}}\right) \mathrm{f}^{2}-\left(\mathrm{H}_{\mathrm{a}}^{2} \sin ^{2} \alpha+\mathrm{D}_{\mathrm{a}}\right) \\
\mathrm{f}^{\prime}+\mathrm{G}_{\mathrm{r}} \theta+\mathrm{G}_{\mathrm{c}} \phi=0 \\
-\mathrm{p}_{\mathrm{d}}^{\prime}=\mathrm{f}^{\prime \prime}+\mathrm{ff}^{\prime}
\end{gathered}
$$

$$
\begin{gathered}
\left(1+\frac{4}{3} \mathrm{R}\right) \theta^{\prime \prime}+\mathrm{P}_{\mathrm{r}} \mathrm{f} \theta^{\prime}-\mathrm{P}_{\mathrm{r}} \mathrm{f}^{\prime} \theta+\mathrm{P}_{\mathrm{r}} \mathrm{Q} \theta=0 \\
\phi^{\prime}+\mathrm{S}_{\mathrm{c}} \mathrm{f} \phi^{\prime}-\mathrm{S}_{\mathrm{c}} \mathrm{f}^{\prime} \phi-\mathrm{S}_{\mathrm{c}} \lambda \phi=0
\end{gathered}
$$

The corresponding initial and boundary conditions take the form:

$$
\begin{aligned}
& \mathrm{f}=\mathrm{f}_{\mathrm{w}}, \mathrm{f}^{\prime}=0, \mathrm{p}_{\mathrm{d}}=0, \theta=1, \phi=1 \text { at } \eta=0 \\
& \mathrm{f}^{\prime}=0, \theta=0, \phi=0 \text { as } \eta \rightarrow \infty
\end{aligned}
$$

Integrating, Eq. 27 with the initial and boundary conditions when $f_{w}=1$ let pressure drop $-p_{d}=G$, this become:

$$
G=f^{\prime}+\frac{1}{2} f^{2}-\frac{1}{2}
$$

Applying WRM to Eq. $26-29$ by assuming a polynomial of with unknown coefficients or parameters to be determined later, this polynomial is called the trial function:

$$
f(\eta)=\sum_{i=0}^{n} a_{i} \eta^{i}, \quad \theta(\eta)=\sum_{i=0}^{n} b_{i} \eta^{i}, \phi(\eta)=\sum_{i=0}^{n} c_{i} \eta^{i}
$$

Impose the boundary conditions (Eq. 28) on the trial functions also substituting the trial functions into Eq. 24,26 and 27 to obtain the residual equations. Minimizing the residual error by forcing it to zero at some set of collocation points within the domain in order to obtain the unknown coefficients using Maple 2016 software:

$$
\begin{aligned}
f(\eta)= & 1.000000000+1.352521223 \eta^{2}- \\
& 2.136719818 \eta^{3}+2.081355570 \eta^{4}- \\
& 1.497172413 \eta^{5}+0.8258367187 \eta^{6}- \\
& 0.3501466456 \eta^{7}+0.1122038542 \eta^{8}- \\
& 0.02624357625 \eta^{9}+0.004215585400 \eta^{10}- \\
& 0.0004145534793 \eta^{11}+ \\
& 0.00001876803320 \eta^{12}
\end{aligned}
$$

$$
\begin{aligned}
\theta(\eta)= & 1.000000000-0.5182224615 \eta+ \\
& 0.003827280553 \eta^{2}+0.1483752625 \eta^{3}- \\
& 0.1835687527 \eta^{4}+0.1537432440 \eta^{5}- \\
& 0.09560654092 \eta^{6}+0.04481892093 \eta^{7}-
\end{aligned}
$$




$$
\begin{aligned}
& 0.01564765871 \eta^{8}+0.003938812348 \eta^{9}- \\
& 0.0006738700894 \eta^{10}+0.00006996577184 \eta^{11}- \\
& 0.000003320194742 \eta^{12} \\
& \phi(\eta)=1.000000000-1.181989422 \eta+ \\
& \quad 0.5262268881 \eta^{2}+0.09051904287 \eta^{3}- \\
& \quad 0.3607131506 \eta^{4}+0.3626303004 \eta^{5}- \\
& \quad 0.2451914515 \eta^{6}+0.1220539007 \eta^{7}- \\
& 0.04472607833 \eta^{8}+0.01171007571 \eta^{9}- \\
& 0.002067978267 \eta^{10}+0.0002202548424 \eta^{11}- \\
& 0.00001066883171 \eta^{12}
\end{aligned}
$$

Differentiate Eq. 31 to obtain:

$$
\begin{aligned}
f^{\prime}(\eta)= & 2.705042446 \eta-6.410159454 \eta^{2}+ \\
& 8.325422280 \eta^{3}-7.485862065 \eta^{4}+ \\
& 4.955020312 \eta^{5}-2.451026519 \eta^{6}+ \\
& 0.8976308336 \eta^{7}-0.2361921862 \eta^{8}+ \\
& 0.04215585400 \eta^{9}-0.004560088272 \eta^{10}+ \\
& 0.0002252163984 \eta^{11}
\end{aligned}
$$

Also, substituting for $\mathrm{f}$ and $\mathrm{f}^{\prime}$ in Eq. 29 with the corresponding constant values to obtain the pressure drop as:

$$
\begin{aligned}
G(\eta)=- & 0.5000000000+2.705042446 \eta- \\
& 6.410159454 \eta^{2}+8.325422280 \eta^{3}- \\
& 7.485862065 \eta^{4}+4.955020312 \eta^{5}- \\
& 2.451026519 \eta^{6}+0.8976308336 \eta^{7}- \\
& 0.2361921862 \eta^{8}+0.04215585400 \eta^{9}- \\
& 0.004560088272 \eta^{10}+0.0002252163984 \eta^{11}+ \\
& \frac{1}{2}\left(1.000000000+1.357521223 \eta^{2}-\right. \\
& 2.136719818 \eta^{3}+2.081355570 \eta^{4}- \\
& 1.497172413 \eta^{5}+0.8258367187 \eta^{6}- \\
& 0.3501466456 \eta^{7}+0.1122038542 \eta^{8}- \\
& 0.02624357625 \eta^{9}+0.004215585400 \eta^{10}- \\
& \left.0.0004145534793 \eta^{11}+0.00001876803320 \eta^{12}\right)^{2}
\end{aligned}
$$

The physical quantity of practical interest are the local skin friction $\mathrm{C}_{\mathrm{f}}$, the Nusselt number $\mathrm{N}_{\mathrm{u}}$ and the local Sherwood number Sh define as:

$$
C_{\mathrm{f}}=\frac{\tau_{\mathrm{w}}}{\rho u_{\mathrm{w}}^{2}}, \mathrm{Nu}=\frac{\mathrm{q}_{\mathrm{w}} \mathrm{x}}{\mathrm{k}\left(\mathrm{T}_{\mathrm{w}}-\mathrm{T}_{\infty}\right)}, \mathrm{Sh}=\frac{\mathrm{q}_{\mathrm{m}} \mathrm{x}}{\mathrm{D}\left(\mathrm{C}_{\mathrm{w}}-\mathrm{C}_{\infty}\right)}
$$

where, $k$ is the thermal conductivity of the fluid, $\tau_{w}, q_{w}$ and $\mathrm{q}_{\mathrm{m}}$ are respectively given by:

$$
\tau_{w}=-\mu\left(\frac{\partial u}{\partial y}\right)_{y=0}, q_{w}=-k\left(\frac{\partial T}{\partial y}\right)_{y=0}, q_{m}=-D\left(\frac{\partial C}{\partial y}\right)_{y=0}
$$

Therefore, the local skin friction coefficient, local Nusselt number and local Sherwood number are:

$$
C_{\mathrm{f}} \operatorname{Re}_{\mathrm{x}}^{\frac{1}{2}}=-\mathrm{f}^{\prime \prime}(0), \mathrm{NuRe}_{\mathrm{x}}^{\frac{1}{2}}=-\theta^{\prime}(0), \operatorname{ShRe}_{\mathrm{z}}^{\frac{1}{2}}=-\phi^{\prime}(0)
$$

where, $\operatorname{Re}_{\mathrm{x}}=v_{\mathrm{w}} \mathrm{X} / \mathrm{vi}$ s the Reynolds number. The process of weighted residual method are repeated for different values of $G_{r}, G_{c}, H_{a} \alpha, Q, P_{r}, D_{a}, R, F_{s} S_{c}$ and $\lambda$. The following computational results in the tables were obtained and compared with Runge-Kutta method generated by maple software.

\section{RESULTS AND DISCUSSION}

In order to get clear insight of the physical problem, numerical computations has been carried out using the method of Weighted Residual for the representative velocity field, pressure field, temperature field, concentration field, coefficient of the skin friction, the rates of heat and mass transfer in terms of Nusselt number and Sherwood number, respectively at the plate have been carried out for different values of the parameters. The following default parameter values are adopted for computation: $\mathrm{G}_{\mathrm{r}}=\mathrm{G}_{\mathrm{c}}=5, \mathrm{Q}=\lambda=0.5, \mathrm{R}=\mathrm{F}_{\mathrm{s}}=\mathrm{D}_{\mathrm{a}}=1, \mathrm{P}_{\mathrm{r}}=$ $0.72, \mathrm{~S}_{\mathrm{c}}=0.62$ and $\mathrm{M}=3$ at $\mathrm{H}_{\mathrm{a}}=6$ and $\alpha=30^{\circ}$. All graphs, therefore, correspond to these values unless specifically indicated on the appropriate graph.

Figure 2 and 3 bring out clearly the effect of the Hartmann number $\mathrm{H}_{a}$ on the velocity and pressure

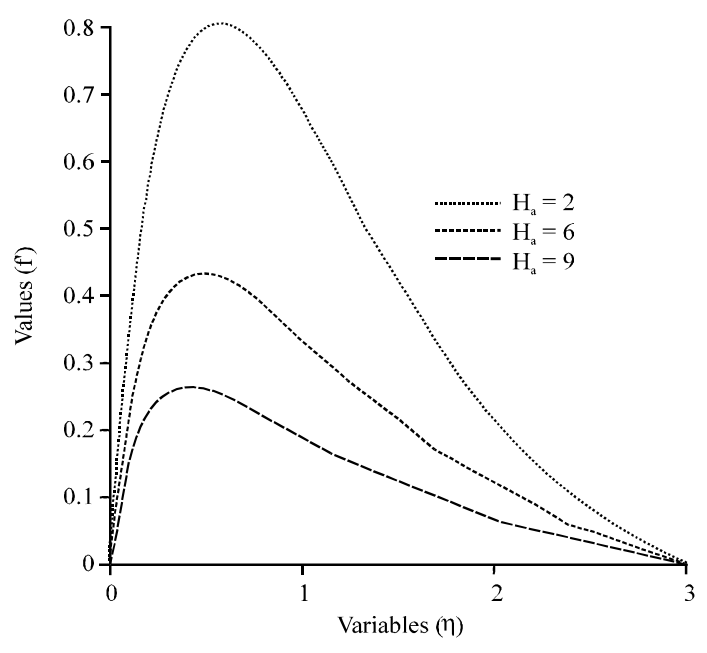

Fig. 2: Velocity profiles for different values of $\mathrm{H}_{\mathrm{a}}$ 


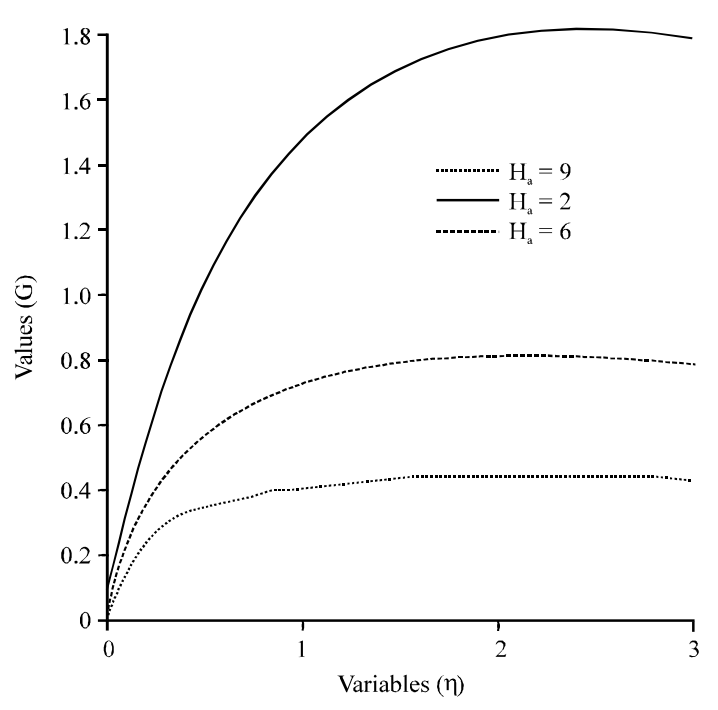

Fig. 3: Pressure profiles for different values of $\mathrm{H}_{\mathrm{a}}$

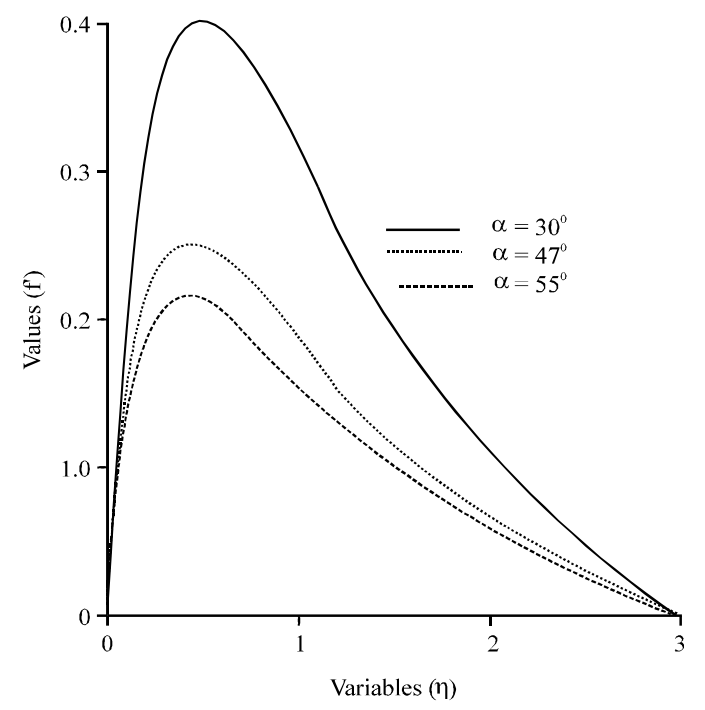

Fig. 4: Velocity profiles for different values $\alpha$

boundary layer thickness, since, the magnetic field exerts a retarding force on the free convection fluid flow. Increasing $\mathrm{H}_{\mathrm{a}}$ decreases the velocity and pressure boundary layer.

Figure 4 and 5 show the velocity and pressure profiles for different angles of inclination of the magnetic field $\alpha$, an increase in the angle of inclination increases the effect of the buoyancy force and consequently, the driving force to the fluid flow decreases as a result, velocity and pressure boundary layer thickness decreases.

Figure 6-9 show the effect of the porosity parameter $\mathrm{D}_{\mathrm{a}}$ and inertial parameter $\mathrm{F}_{5}$ on the velocity and pressure profiles. It was observed that the velocity and pressure

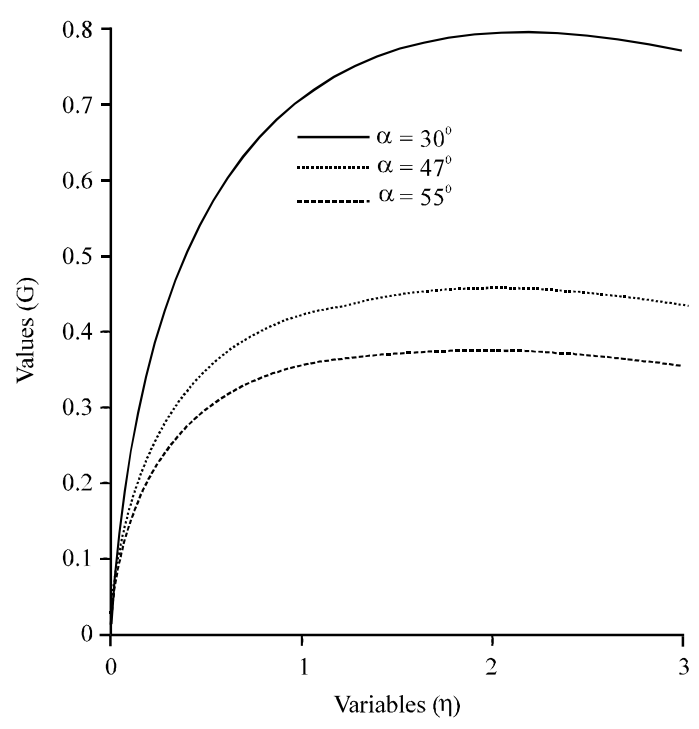

Fig. 5: Pressure profiles for different values $\alpha$

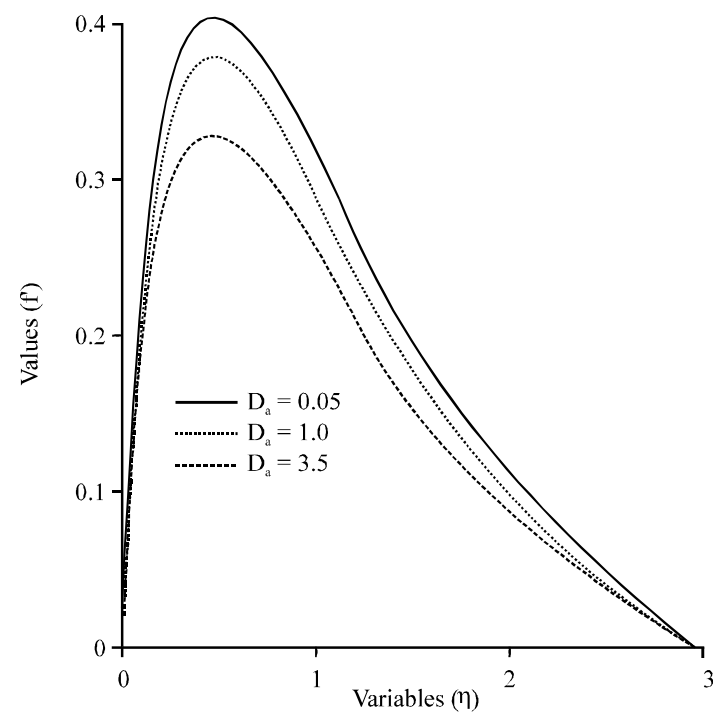

Fig. 6: Velocity profiles for different values $D_{a}$

decreases as the porosity and inertial parameter increases. The reason for this behavior is that the wall of the surface provides an additional resistance to the fluid flow mechanism which causes the fluid to move at a retarded rate.

Figure 10 and 11 depict the influence of different values of the Prandtl number $P_{r}$ on the pressure and temperature distribution. It is observed that an increase in the ratio of momentum diffusivity to thermal diffusivity results in the, respectively decrease in pressure and temperature profiles. The reason for this behavior is that an increase in the $\mathrm{P}_{\mathrm{r}}$ results in a decrease in the boundary layer thickness and reduce the average temperature within the boundary layer because smaller values of $P_{r}$ are 


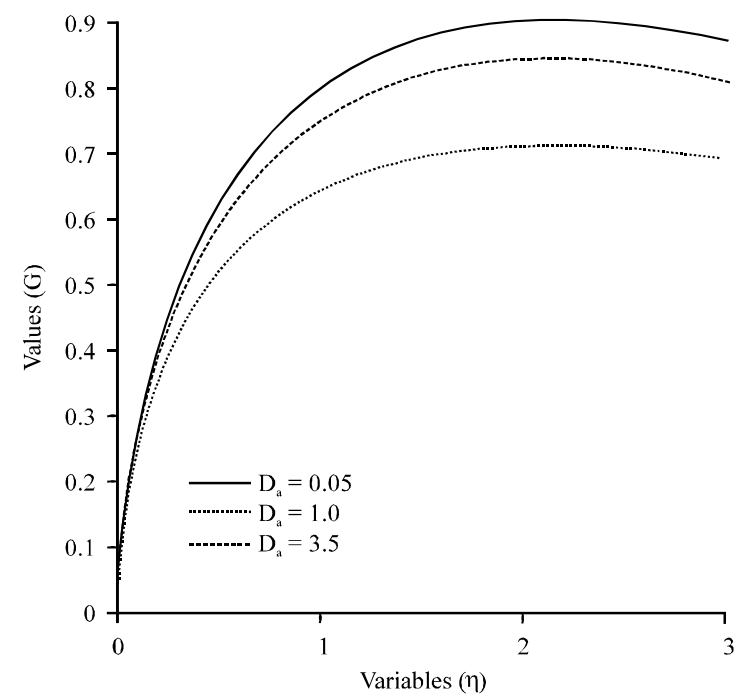

Fig. 7: Pressure profiles for different values of $D_{a}$

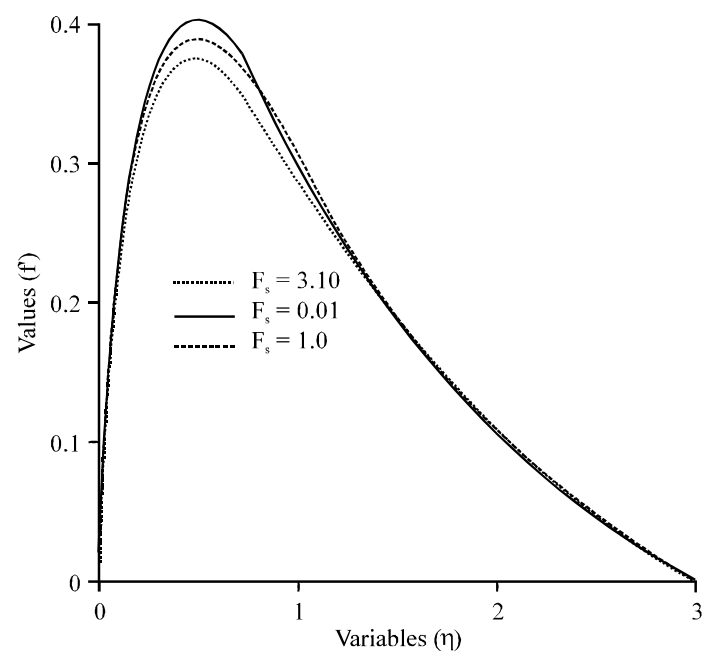

Fig. 8: Velocity profiles for different values of $F_{s}$

equivalent to increasing in the thermal conductivities, therefore, heat is able to diffuse away from the heated plate than higher values of $P_{r}$.

The effect of Schmidt number $S_{c}$ on the pressure and concentration profiles are represented on Fig. 12 and 13. Schmidt number is the ratio of the momentum to the mass diffusivity. An increase in $S_{c}$ causes reductions in the pressure and concentration profiles which are accompanied by simultaneous decrease in the pressure and concentration boundary layers. Schmidt number is therefore, quantifies the relative effectiveness of momentum and mass transport by diffusion in the hydrodynamic pressure and concentration boundary layers. All these agrees with the expectations.

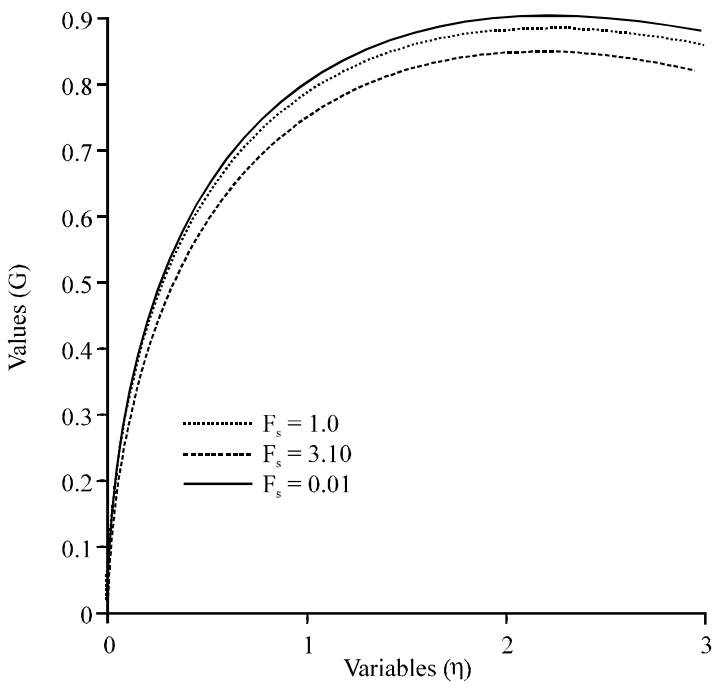

Fig. 9: Pressure profiles for different values of $F_{s}$

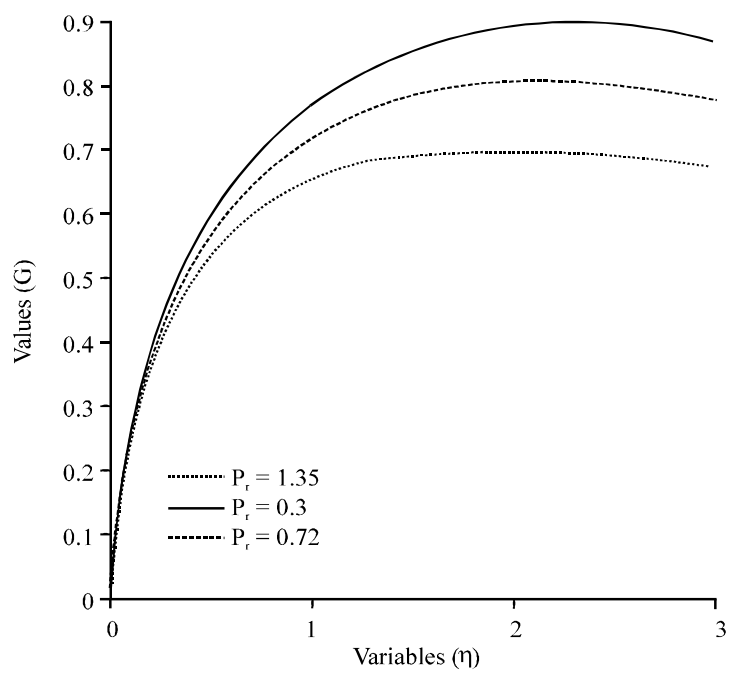

Fig. 10: Pressure profiles for different values of $P_{r}$

Table 1 depicts the effect of some physical parameters on skin friction, nusselt and Sherwood number. It clearly shows that, the magnetic term $\mathrm{H}_{a}$ and angle of inclination $\alpha$ have a retarding effect on the skin friction, nusselt and Sherwood number, respectivly. Increase in heat source increase skin friction and Sherwood number while it decreases the Nusselt number because heat within the boundary layer reduces. Also, number Prandtl number $P_{\mathrm{r}}$ has decreasing effect on the skin friction and Sherwood number but has an increasing effect on Nusselt number because of the thickness of the thermal boundary layer. Moreover, skin friction and nusselt have a decelerating effect on increasing Schmidt number but an increasing effect on increasing Schmidt number. 
Table 1: Effect of $\mathrm{H}_{a}, \alpha, Q, \mathrm{P}_{r}, \mathrm{~S}_{\mathrm{c}}$ on $\tau, \mathrm{Nu}$ and Sh (PP-Phy sical Parameters)

\begin{tabular}{|c|c|c|c|c|c|c|c|}
\hline \multirow[b]{2}{*}{ PP } & \multirow[b]{2}{*}{ Values } & \multicolumn{3}{|c|}{ Weighted Residual method } & \multicolumn{3}{|c|}{ 4th order R-K } \\
\hline & & $\tau$ & $\mathrm{Nu}$ & Sh & $\tau$ & $\mathrm{Nu}$ & Sh \\
\hline \multirow[t]{3}{*}{$\overline{\mathrm{H}_{\mathrm{a}}}$} & 2 & 4.05305 & 0.64028 & 1.31465 & 4.05634 & 0.64017 & 1.31431 \\
\hline & 6 & 2.70504 & 0.51822 & 1.18199 & 2.71947 & 0.51806 & 1.18134 \\
\hline & 9 & 2.00826 & 0.45420 & 1.10920 & 1.98953 & 0.45406 & 1.10844 \\
\hline \multirow[t]{3}{*}{$\alpha$} & $30^{\circ}$ & 2.70504 & 0.51822 & 1.18199 & 2.71947 & 0.51807 & 1.18134 \\
\hline & $47^{0}$ & 2.05511 & 0.45787 & 1.11345 & 2.02117 & 0.45772 & 1.11269 \\
\hline & $55^{0}$ & 1.86538 & 0.44189 & 1.09484 & 1.82722 & 0.44175 & 1.09408 \\
\hline \multirow[t]{3}{*}{ Q } & 0.5 & 2.70504 & 0.51822 & 1.18199 & 2.71947 & 0.51806 & 1.18134 \\
\hline & 0.8 & 2.74628 & 0.42937 & 1.18688 & 2.74405 & 0.42923 & 1.18622 \\
\hline & 1.2 & 2.78445 & 0.29558 & 1.19442 & 2.79831 & 0.29547 & 1.19377 \\
\hline \multirow[t]{3}{*}{$\mathrm{P}_{\mathrm{r}}$} & 0.3 & 2.75338 & 0.40518 & 1.19053 & 2.76763 & 0.40513 & 1.18987 \\
\hline & 0.72 & 2.70504 & 0.51822 & 1.18199 & 2.71947 & 0.51806 & 1.18134 \\
\hline & 1.35 & 2.63053 & 0.71094 & 1.16950 & 2.64527 & 0.71056 & 1.16886 \\
\hline \multirow[t]{3}{*}{$S_{c}$} & 0.4 & 2.79921 & 0.52961 & 0.90598 & 2.79305 & 0.52945 & 0.90565 \\
\hline & 0.62 & 2.705042 & 0.518222 & 1.181989 & 2.71947 & 0.51806 & 1.18134 \\
\hline & 1.5 & 2.472478 & 0.493724 & 2.173529 & 2.48940 & 0.49357 & 2.17044 \\
\hline
\end{tabular}

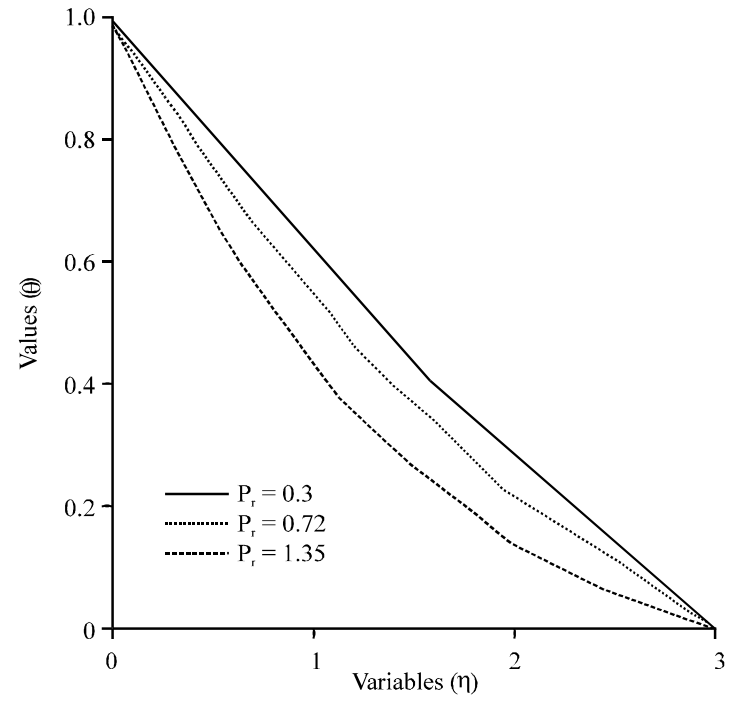

Fig. 11: Temperature profiles for different values of $P_{r}$

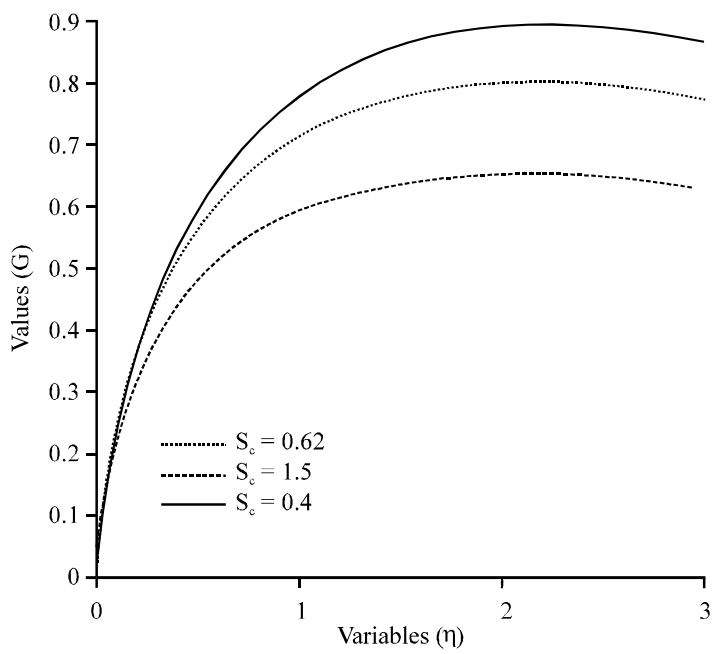

Fig. 12: Pressure profiles for different values of $\mathrm{S}_{c}$

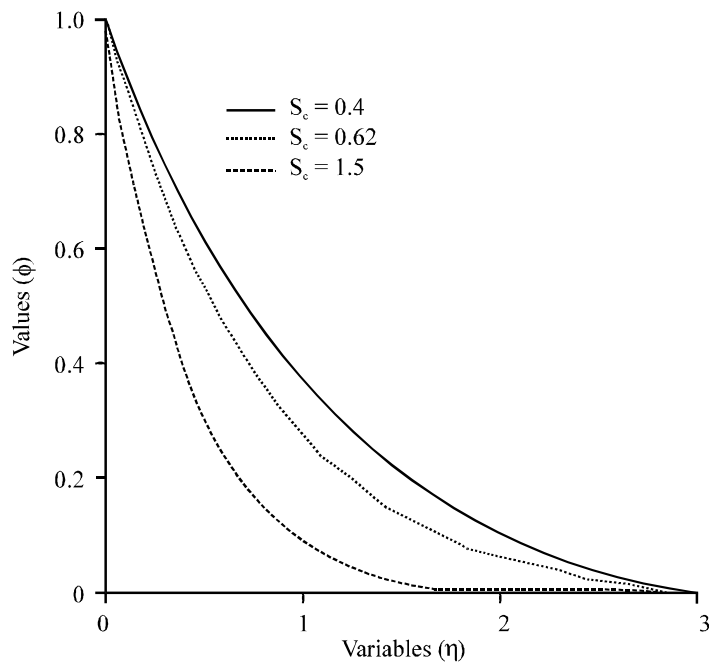

Fig. 13: Concentration profiles for different values of $S_{c}$

\section{CONCLUSION}

The non-dimensional of the formulated governing equations are reduced to a couple ordinary differential equations by using scaling group transformation. The numerical solution are obtained using Weighted Residual method. From the numerical results, it is seen that, an increase in the values of Hartmann, degree of inclination of the magnetic field, porosity parameter, inertial parameter, Prandtl or Schmidt numbers is manifested as a decrease in the flow velocity, pressure, temperature and concentration distribution.

The results of the study are interested because flows past a permeable surface play an essential role in applications of science and engineering, also in many transport processes in nature. 


\section{REFERENCES}

Afify, A.A. and N.S. Elgazery, 2012. Lie group analysis for the effects of chemical reaction on $\mathrm{MHD}$ stagnation-point flow of heat and mass transfer towards a heated porous stretching sheet with suction or injection. Nonlinear Anal. Modell. Control, 17: $1-15$.

Ahammad, M.U., M. Obayedullah and M.M. Rahman, 2013. Analysis of MHD free convection flow along a vertical porous plate embedded in a porous medium with magnetic field and heat generation. Eng. Trans., 8: 10-18.

Ahmed, N. and D.J. Bhattacharyya, 2014. Free convection in $\mathrm{MHD}$ couette flow with heat source and chemical reaction. Appl. Math. Sci., 8: 2473-2482.

Alam, M.S., S.M.C. Hossain and M.S.H. Mollah, 2003. MHD free convective flow along an inclinedpermeable stretching sheet with viscous dissipation and chemical reaction. Intl. J. Res. Manage., 1: 1-13.

Chauhan, D.S. and P. Rastogi, 2010. Radiation effects on natural convection $\mathrm{MHD}$ flow in a rotating vertical porous channel partially filled with a porous medium. Appl. Math. Sci., 4: 643-655.

Dada, M.S. and F.H. Adefolaju, 2012. Dissipation, $\mathrm{MHD}$ and radiation effects on an unsteady convective heat and mass transfer in a darcy-forcheimer porous medium. J. Math. Res., 4: 110-127.

Hemalatha, K., M.V.D.N.S. Madhavi and D. Rajani, 2014. Melting with thermal dispersion on non-darcy MHD mixed convective flow from a vertical plate embedded in a saturated porous medium. Intl. J. Math. Stat. Invention, 2: 19-27.
Hunegnaw, D. and N. Kishan, 2014. Unsteady MHD heat and mass transfer flow over stretching sheet in porous medium with variable properties considering viscous dissipation and chemical reaction. Am. Chem. Sci. J., 4: 901-917.

Ishak, A. and R. Nazar, 2006. Steady and unsteady boundary layers due to a stretching vertical sheet in a porous medium using darcy-brinkman equation model. Intl. J. Appl. Mech. Eng., 11: 623-637.

Kareem, R.A., S.O. Salawu and J.A. Gbadeyan, 2018. Numerical analysis of Non-uniform heat source/sink in a radiative micropolar variable electric conductivity fluid with dissipation joule heating. Am. J. Appl. Math., 6: 34-41.

Kumar, B.R. and R. Sivaraj, 2012. MHD viscoelastic fluid non-darcy flow along a moving vertical cone. Intl. J. Appl. Math. Mech., 8: 69-81.

Lee, J., P. Kandaswamy, M. Bhuvaneswari and S. Sivasankaran, 2008. Lie group analysis of radiation natural convection heat transfer past an inclined porous surface. J. Mech. Sci. Technol., 22: 1779-1784.

Loganathan, P. and P.P. Arasu, 2010. Thermophoresis effects on non-darcy $\mathrm{MHD}$ mixed convective heat and mass transfer past a porous wedge in the presence of suction/injection. Theor. Appl. Mech., 37: $203-227$.

Pramanik, S., 2013. Applications of scaling group of transformations to the boundary layer flow of a non-newtonian power-law fluid. Intl. J. Appl. Math Mech., 9: 1-13.

Saxena, S.S. and G.K. Dubey, 2011. Unsteady MHD heat and mass transfer free convection flow of polar fluids past a vertical moving porous plate in a porous medium with heat generation and thermal diffusion. Adv. Appl. Sci. Res., 2: 259-278.

Singh, G., P.R. Sharma and A.J. Chamkha, 2012. Mass transfer with chemical reaction in MHD mixed convective flow along a vertical stretching sheet. Intl. J. Eng. Technol., 4: 1-12. 